\title{
International Norms and Socio-technical Systems: Connecting Institutional and Technological Infrastructures in Governance Processes*
}

\author{
Claudia Padovani ${ }^{1}$ and Elena Pavan ${ }^{2}$ \\ ${ }^{1}$ Dipartimento di Scienze Politiche, Giuridiche e Studi Internazionali, Uni. of Padova, Italy \\ claudia.padovani@unipd.it \\ ${ }^{2}$ Dipartimento di Sociologia e Ricerca Sociale, University of Trento, Italy \\ elena.pavan@unitn.it
}

\begin{abstract}
This paper looks at the challenges posed by ICT critical infrastructures in their interaction with governance processes. The authors argue that, in order to develop better understanding of how (global) governing arrangements are made in a highly mediatised environment, adequate frameworks should be elaborated to study the interrelation between institutional and technological infrastructures. In this context, institutions are conceived as collections of norms including a mix of rules and practices - while technological infrastructures are seen as instruments that transform governance processes, also enabling different actors' participation. Adopting a constructivist approach, combined with a focus on governance networks, the authors introduce a multi-dimensional analytical framework to investigate governance processes where institutions and technologies converge to create socio-technical systems.
\end{abstract}

Keywords: international norms, socio-technical systems, institutional and technological infrastructures, communication governance, analytic framework.

\section{Introduction}

There are at least three ways in which we can read the 'non-neutrality' of technology - and of ICT in particular. First, from a broad social perspective, ICT inform and reform the dynamics of con-temporary societies constituting one of the primary elements for globalizing processes [5], [12]: they transform societal interactions, respond to and solicit needs, create expectations. Hence, in a first sense, ICT are not neutral because they are mediating infrastructures that contribute to change both societal perceptions of the world and of the role of human agency in it.

\footnotetext{
* The authors share responsibility for the contents of this article and jointly realized the Introduction and sections $\S 2.3, \S 3$ and $\S 4$. However, they contributed to the realization of specific sections. In particular, $\$ 2.1$ was drafted by Claudia Padovani; $\$ 2.2$ was drafted by Elena Pavan in conjunction with the project REACtION, funded by the Provincia Autonoma di Trento (PAT) and carried on at the University of Trento, Department of Sociology and Social Research (www.reactionproject.info).
} 
Secondly, from a political perspective, ICT have progressively emerged as a policy relevant domain [22]. In the last 50 years or so, from the national to the global level, policy actors have been called to respond to challenges posed by technological transformations. Moreover, the consolidation of the ICT policy field has been accompanied by the evolution of a number of different governing arrangements - selfgovernance, public-private partnership, multi-stakeholder interventions - aimed at meeting the challenges brought by technological development to regulatory practices. Hence, in a second way, technology is not neutral because it constitutes a domain wherein different political interests and agendas are played out and because it has fostered change in (especially supra-national) governance practices.

Thirdly, at the junction of the social and the political perspectives, ICT evolution (and particularly of the Internet) and their capillary penetration into our daily lives do multiply the possibility for individuals, groups, communities to act politically and become 'part of the process.' As ICT contribute to the structuring of network societies, they also challenge traditional political processes by enabling political participation of non-conventional and non-governmental actors. This also favours the diffusion of ideas, knowledge, and cultural practices that characterize such diverse sets of actors. Hence, in a third way, ICT are not neutral because they become enabling factors for citizens' involvement, both as individuals and in their associational forms.

While social transformations brought about by ICT developments and diffusion have received increased scholarly attention, we are still in need of adequate conceptual tools and analytical frameworks to investigate the dynamics that develop when transformed policy arrangements meet the opportunity and claims for citizens' enhanced participation. This includes frameworks to investigate both the basic principles that guide the development of technological infrastructures, but also the principles those very technologies may contribute to shape and consolidate, including in other domains beside and beyond technology governance. Adequate frameworks should then be elaborated to study the interrelation between institutional and technological infrastructures. Institutions are to be understood here as collections of norms, including a mix of rules and practices [8]; while technological infrastructures are seen simultaneously as instruments for global interaction, means for transforming (global) governance processes, and enabling factors for participation in political processes.

In our attempt to address this challenge, we believe a constructivist approach to world politics, provides a fruitful theoretical framework within which to position our reflections. Such an approach is grounded in the conviction that actors' interpretations of the material world depends on dynamic epistemic and normative understandings [19]: the world is not a given, but rather the outcome of interactions and interpretations based on actors' experience, knowledge, and preferences. In this context, ideational forces - ideas, values, knowledge - become central and imbue communicative exchanges [14] that sometimes consolidate into discourses, understood as "shared sets of ideas that provide frameworks that allow us to make sense of situations, embody judgments, and foster capabilities" [7].

Starting from these premises, we argue that a focus on the ideational components of policy processes - and particularly norms - is crucial in studying the interrelationships between institutional and technological infrastructures. Indeed, norms emerge at 
the intersection between actors' discursive interactions, through which they may redefine their identities and interests. As "shared standards of behaviour for actors with a given identity," [8] norms represent the consolidation of principled discourses into defined frameworks. Hence, agreed upon normative frameworks inform and orientate the outcome of political processes; consequently, they can be understood as one of the core 'infrastructural' elements (beside formal rules and provisions) that support governance processes and subsequent policy decisions.

Moreover, the emergence and consolidation of norms presupposes the interplay of cognitive and relational components upon which the current development and diffusion of ICT has a dramatic impact. In fact, the unprecedented levels at which ICT are embedded into every domain of human action fosters what we label socio-technical breakthrough: a unique situation where the social and the technical infrastructures overlap and create a hybrid social space where the circulation of ideas and principles, and the construction of social relations, cross the boundary between 'the virtual' and 'the real' thus making the distinction between the online and the offline obsolete. This breakthrough poses new challenges to the study of normative developments, and invites us to expand our perspective to include in the analysis all those practices - framing, agenda setting - that inform norms' consolidation beyond the translation into official provisions, as well as the technical infrastructures that accompany and support such practices.

This paper acknowledges this centrality of norms and outlines an analytical approach to the study of the relationship between institutional and technological infrastructures. Our approach aims at meeting the conceptual and methodological challenges posed by the current socio-technical context to the study of normative frameworks emergence and consolidation, in particular that of overcoming the separation between the online and the offline. Also, our approach translates into an analytic framework whose components can be flexibly adapted to different governance domains.

The paper is organized as follows. First, we outline the two core conceptual elements of our proposal: the study of norms as 'infrastructures' in world politics and the broader context in which they evolve, which we understand as a set of 'sociotechnical systems.' We conclude this first section outlining the methodological challenges to be addressed. Afterwards, we introduce and illustrate an analytical framework elaborated to meet the identified methodological and theoretical challenges. We conclude by discussing research perspectives in the application of the framework and we outline the deriving implications by referring to an ongoing research project.

\section{Core Elements and Methodological Challenges}

\subsection{Norms as 'Infrastructures’ of Governing Arrangements}

Global governance can be understood as the multiplicity of networks of interdependent, but operationally autonomous actors, that produce relevant knowledge and cultural practices and/or develop frames that imbue public discourse and orientate policy 
agendas and/or articulate principles, norms and rules while engaging in political negotiation with a view to orientate policy-relevant outcomes [22], [20].

As a specific ideational element in the dynamics we observe, norms set standards of behaviour for actors; for instance, contributing to the definition of limits to stateactors' agency in fostering national interests and committing states and other actors to respect agreed upon principles and fundamental rights. Norms emergence and evolution on the supra-national scene are, therefore, central to governance processes, as they open up spaces for change in actors' preferences and provide reference to appropriate behaviour, sometimes contributing to "restructuring world politics" [15].

We, therefore, argue that norms should not be conceived of as 'soft' components of political processes, in comparison to the 'hard' nature of regulation and decisionmaking. Rather, norms can be considered as 'infrastructures' that enable and structure such processes. Norms contribute to the definition and transformation of actors' identities and perceptions, and to orientate their behaviour. Norms also provide cognitivenormative spaces for the formulation of new policies [23]. As a consequence, their definition may become a site of struggle for actors who strategically engage in 'struggles over meaning'; thus, norms - as agreed upon standards of behaviour - can also be understood as a reflection of the (structure of) strategic interactions and power struggles that occur in political processes ${ }^{1}$.

Several authors remind us that norms are "difficult to see" (when do we recognize a norm when we see one?); and yet "leave an extensive trail of communication among actors that we can study" [8]. For example, we can investigate statements and policy inputs as well as less formalized discursive practices amongst actors. In doing so, it is possible to trace their "life-cycle" of "emergence" in the policy context, including the following processes: "cascade" occurs when their adoption by a wide number of relevant actors turns them into "standards of behaviour"; while "internalization" occurs when they become "a given" and are perceived as natural frameworks to orientate actors' behaviour [8], [9].

More recent literature on norms suggests a more articulated view of such a 'lifecycle' [15], [23], [17], according to which norms are always contested because, even after adoption by a broad number of actors, struggles may emerge between alternative perspectives. Furthermore, norms do not automatically imply change; rather, they provide opportunities for actors' behaviour and, as such, they are always 'works in progress' in that they are constructed and re-constructed in practice, according to actors' adoption in their actual activities and political interventions. Therefore, looking at international norms calls for a specific attention to the multi-level 'game' of interaction between norms adoption and application at the local/national and at the global levels [4]. Finally, norms are always exposed to 'shocks': changes in the envi-

\footnotetext{
${ }^{1}$ As a consequence, norms and normative frameworks can also be investigated in relation to the different forms of power that intervene in their very elaboration and adoption: the productive power of issue framing, the institutional power of agenda setting, the coercive power of norms definition and application, as well as the network power of actors engaging in normoriented interactions. Limited space here does not allow an adequate elaboration on the challenges of adopting a multi-dimensional approach to power in world politics and global governance; we refer to the relevant literature [1], [3], [6].
} 
ronment where norms are debated, applied, and contested can certainly interfere with their consolidation and eventually lead to further redefinition of these normative infrastructures. This has been the case, in the context of norms pertaining to global communications and its governance, with the explosion, evolution and diffusion of information technologies in the past two decades: a situation that has originated what can now be conceived as 'socio-technical systems.'

\section{$2.2 \quad$ Socio-technical Systems}

It is widely acknowledged that developments in the ICT domain have been one of the driving forces of globalization processes. Hence, the network society we live in is first and foremost a society of communication networks: "patterns of contact that are created by the flow of messages among communicators through time and space" [18]. The fact that ICT are now ubiquitous [11] makes communication networks global in a twofold way: they potentially expand along telecommunication infrastructures to reach all corners of the globe; and, with the diffusion of computers and portable devices, they innervate all fields of public and private action.

Together with Hall [11], we support a vision for which changes in ICT are shaped by and, at the same time, shape society in a fluid and very dynamic way. In this sense, we believe that changes in ICT influence and, at the same time, are influenced by the structure of communication networks that innervate our society. Consistent with this perspective, we also argue that the integration of Internet communication into mobile and portable devices together with the transition to Web 2.0, are by-products of and an influencing factors in relation to the structure and the dynamics of global communication networks. Indeed, the ubiquitous presence of Internet has progressively led to a situation of seamless adaptation of social relations to the Internet infrastructure [10]. As a consequence, the online and the offline dimensions merge together, thus blurring the boundaries between the virtual and the real, and technology becomes the means through which we make sense of our social reality more and more [2].

In such a context, global communication networks resulting from the interconnection of individuals and groups and events and data are better seen in terms of sociotechnical systems that result from the intertwinement of two 'infrastructure': one given by the maze of social relations, the other by the maze of physical networks on top of which they are built [24]. The social space within socio-technical systems is hybrid, as it results from the merging of the online and the offline, while social relations become cross-dimensional because they are nurtured by both mediated and unmediated interactions and are defined recursively across the two spaces [2].

Also, the transition from Web 1.0 to Web 2.0 technologies changes the logic through which connections within socio-technical systems are structured. From a 'culture of publicity' pursued through websites in the Web 1.0 era, we switch now to a 'culture of participation' pursued through social media tools [13]. The key element is the possibility for users to create and share their own information and content in real time through social media platforms, which reduces the need for specific technical knowledge for content creation, meaningfully augmenting the possibilities to enter global communication networks [11]. 


\subsection{The Challenge of Investigating Norms in Socio-technical Environments}

According to what has been exposed, the study of norms evolution within dynamic socio-technical systems can prove to be a useful entry point to a better understanding of the interrelation between institutional and technological infrastructures (in our case in relation to the global governance of communication). If we see norms as 'institutional infrastructures' and socio-technical systems as a revised version of 'technological infrastructure,' we are left with the challenge of developing adequate analytical frameworks to study processes that take place at the junction of policy arrangements and citizens' involvement.

Norms as institutional infrastructures reflect the richness of the ideational components that enter governance processes and embody the interplay of power positions, thus inviting due consideration of how such processes are deployed. We argue that it is often in networked interactions that governing arrangements are being shaped: ideas shared, issues framed and put on the agenda, priorities defined, alternative solutions discussed, and norms agreed upon [20], [21]. And yet, as they operate within socio-technical systems, these networks are constantly at risk of being transformed by available technological infrastructures at least at two levels:

- Ideational/Communicative: The articulation of policy issues and decisions happens on the basis of discursive practices for which both the social interaction amongst actors and the semantics around which interactions evolve are relevant. ICT impact the creation of both social and semantic relations as they increase the relational potential of every actor (individual, organizational, governmental and nongovernmental nature) and fosters the circulation, the blending and the clash of ideas, agendas and perspectives.

- Spatial: The dynamics of social and semantic construction of governance practices are recursive between the off-line (within expert committees, on the occasion of high level summits or less formal gatherings) and the on-line (through on-line consultations and mailing list exchange, but also through the structuring of thematic and issue networks in the web space). ICT embeddedness in daily social and political practices fosters the merging of the two dimensions and the hybridization of practices.

The challenge to be faced in investigating norms in their socio-technical context is to find adequate ways of dealing with the complexities deriving from actors' interactions across diverse ideational as well as spatial levels/spaces. We contend that a focus on actors' characteristics and attributes - such as the human, financial, and symbolic resources they can play out in a policy negotiations - does not suffice in addressing the complexities deriving from multi-level interactions.

\section{Connecting Institutional and Technological Framework}

To investigate the interplay between institutional and technological infrastructures, we propose a multidimensional analytical framework based on the concept of 
networks, which we consider as a suitable metaphor but also as a powerful analytic tool. The framework has been developed for the study of global communication governance but it is proposed here as a resource to investigate other policy domains. In order to address the challenges outlined above, a focus on relational dynamics involving both actors and concepts allows us to trace the 'trails of communication' that underpin the evolution of normative frameworks in governing processes. Acknowledging the "dynamics, diversity, and complexity" of such processes [16], different types of networks provide entry points for investigating meaningful aspects: semantic networks are useful to "map similarities amongst [actors' perceptions and] interpretations" [18], while social networks help unveiling patterns of collaborative and/or conflicting interaction. Moreover, in the context of widely diffused information technologies, semantic as well as social network develop across the online/offline boundary. In response to this, the explicit acknowledgement of both online and offline interactions interactions in our framework provides the means to acknowledge and investigate the hybrid feature of social space within socio-technical structures.

Table 1. Analytic framework to study networks and interactions in governance processes

\begin{tabular}{llll}
\hline & & Spatial Dimension & \\
\hline Ideational/Communicative & Social & Offline & Online \\
Dimension & & Offline Collaboration/ & Online Networks of \\
& Conflicting Networks & Interaction \\
& Semantic & $\begin{array}{l}\text { Offline Semantic } \\
\text { Networks }\end{array}$ & $\begin{array}{l}\text { Online Thematic } \\
\text { Networks }\end{array}$ \\
\hline
\end{tabular}

Although each network type included in Table 1 helps us to understand a specific aspect of normative framework evolution, only by analyzing and interpreting the combination and interplay of the different network structures we can reach a more comprehensive understanding of how policy domains are structured, where their boundaries are set, and what kinds of power are distributed along network ties and among actors. We have elaborated elsewhere on the heuristic potential of a network approach to communication governance [20]; here we provide a brief description of what each network allows us to look at and investigate.

Offline collaboration/conflicting networks gather social actors - whether they are individuals or organizations - operating in a certain policy domain. They can be read in terms of how relational patterns are being developed in a multi-actor environment; thus clarifying what logics inform political interactions, such as long term solidarity and instrumental coalition-building. Indeed, ties between actors exist if a particular kind of relationship is established amongst nodes, such as cooperation, opposition, or sponsorship; and relationships can be characterized by presence, absence, and strength, such as quantifying the number of joint initiatives two organizations have realized. Furthermore, the specific positions of nodes can be evaluated in terms of their prestige, whether they involve a brokerage role, or the facilitation or control of communication flows within the network. 
Offline semantic networks trace conversational patterns along which different themes are brought into the policy agenda and, in this way, provide a useful entry point from which to assess how discursive practices actually inform political processes, possibly influencing policy outcomes. They depict the collective construction of meanings and are particularly relevant when it comes to analyzing governance structures that may not aim at producing formal policy outputs, such as high-level summits as occasions for multi-stakeholder debates. Nodes are concepts and the presence of ties can be understood as a positive association among issues; while the absence of ties signals a disconnection in conversational dynamics. Also, the strength of a tie can be conceived in terms of 'semantic proximity' between different themes: the stronger the tie, the greater the association between two issues. Also, more central themes indicate priorities emerging in a field, while clusters can be read in terms of subfields catalyzing the attention of specific actors.

Online networks of interaction represent conversational dynamics deployed online that often, but not necessarily, accompany more formal governance processes. Analyzing this type of network can help uncover dynamics that may create the conditions for an enlargement or refinement of the policy agenda when physical presence is not possible. These networks can be structured by more participative settings, such as conversations involving several actors; less shared exchanges, such as conversations between only two actors; and information sharing, such as messages sent where no response is required. Nodes can be actors entering a specific discursive space, such as a forum, a mailing list; but they can also be web pages, web portals, or news services relevant to the issues being addressed. Ties can be traced to investigate actors' participation and this would allow for the identification of 'hot-spots' of online discussions. Also, ties could be traced to indicate participation in the same conversational thread, thus contributing to the identification of 'hot-topics' in the same discussions.

Online thematic networks show how different web-based resources, such as websites, online documents, and blogs, deal with policy areas that are related to one another through hypertext links. Thus online thematic networks may provide insights on how multi-actor conversations pushed offline by a relevant governance process are translated into web-based conversational fluxes that are accessible to all Internet users. Thematic networks can also help in mapping out the online spaces of discourse that parallel social mobilization around specific issue areas or topics. These networks can be made up of very heterogeneous nodes connected by a relation of recognition, which are not necessarily mutual; and their analysis contributes to assessing how a diversified articulation of relevant discourses may take place in the web-sphere.

\section{$4 \quad$ Research Perspectives and Framework Application}

In this paper, we have discussed the interrelationships between institutional and techno-logical infrastructures. Our discussion started from the centrality of international norms as ideational elements that are crucial for governing arrangements; and we considered the challenges posed by growingly mediatised social contexts in which 
norms emerge and evolve that we label 'socio-technical systems.' We then proposed a multidimensional analytical framework to investigate such complex dynamics and address the challenges posed by the consolidation of socio-technical systems. By explicitly including social and semantic as well as online and offline dimensions, the proposed approach allows us to account for the dispersion and multiplication of cognitive and relational elements that characterize the 'life-cycle' of normative frameworks within socio-technical systems. Furthermore, by looking at networked interactions, the approach moves from a predominant focus on the formal diversity of the actors involved in governance dynamics to a better understanding of how different constituencies relate to one another and how such patterns of interaction might change over time, thus providing the grounds for norms evolution.

We are currently testing this approach by applying the framework to the case study of 'gender-oriented communication governance,' understood as that area of global communication governance that pertains to the nexus between media and gender ${ }^{2}$. The ongoing project maps all four types of networks, investigates the position of nodes, and interprets links amongst them in order to trace the evolution of normative frameworks that have over time informed governing arrangements in this domain, from the local to the global. The final aim is to reach a comprehensive understanding of how institutional and technological infrastructures interact and contribute to the transformation of power relations in the domain under investigation.

The actual conduct of the project and preliminary results seem to confirm the validity of the approach, pointing to the necessity for a joint reading of results obtained from the exploration of the different types of networks. Furthermore, it provides elements to critically reflect on the implications of translating the theoretical framework into empirical fieldwork. Certainly, research in different domains will be needed to further assess the heuristic potential of the proposed perspective and methodological approach.

\section{References}

1. Barnett, M., Duvall, R.: Power in International Politics. International Organization 59(1), 39-75 (2005)

2. Beer, D.: Social Network(ing) Sites/Dots Revisiting the Story so far: A Response to Danah boyd \& Nicole Ellison. Journal of Computer-Mediated Communication 13, 516-529 (2008)

3. Berenskoetter, F., Williams, M.J.: Power in World Politics. Routledge, London (2007)

4. Brown Thompson, K.: Women's Rights are Human Rights. In: Khagram, S., Riker, J.V., Sikkink, K. (eds.) Restructuring World Politics: Transnational Social Movements, Networks and Norms, pp. 96-122. University of Minnesota Press, Minneapolis (2002)

${ }^{2}$ Funded by the University of Padova, the project titled 'Networks and Power in Genderoriented Communication Governance,' adopts the theoretical approach and analytical framework outlined in this contribution. The case study has been chosen because it presents most of the features that characterize global governing arrangements, and because, due the long 'history' of supra-national interventions in this area, it allows to trace and compare stages of norms formation that have been characterized by different types of infrastructures, only recently coming to reflect what we have identified as 'socio-technical systems.' 
5. Castells, M.: The Rise of Network Society. Blackwell Publishers, Oxford (1996)

6. Castells, M.: A Network Theory of Power. International Journal of Communication 5, 773-787 (2011)

7. Dryzek, J.S.: Deliberative Democracy in Divided Societies: Alternatives to Agonism and Analgesia. Political Theory 33(2), 218-242 (2005)

8. Finnemore, M., Sikkink, K.: International Norm Dynamic and Political Change. International Organization 52(4), 887-917 (1998)

9. Florini, A.: The Evolution of International Norms. International Studies Quarterly 40(3), 363-389 (1996)

10. Giunchiglia, F., Robertson, D.: The Social Computer: Combining machine and human computation. DISI, University of Trento, Tech. Rep. 10-036 (2010),

http://eprints.biblio.unitn.it/archive/00001851/01/036.pdf

11. Hall, W.: The Ever Evolving Web: The Power of Networks. International Journal of Communication 5, 651-664 (2011)

12. Held, D., McGrew, A., Goldblatt, D., Perraton, J.: Global Transformations: Politics, Economics, and Culture. Polity Press, Cambridge (1999)

13. Kaplan, A.M., Haenlein, M.: Users of the World, Unite! The Challenges and Opportunities of Social Media. Business Horizion 53, 59-68 (2010)

14. Keck, M.E., Sikkink, K.: Activists Beyond Borders: Advocacy Networks in International Politics. Cornell University Press, New York (1998)

15. Khagram, S., Riker, J.V., Sikkink, K. (eds.): Restructuring World Politics. Transnational Social Movements, Networks and Norms. University of Minnesota Press, Minneapolis (2002)

16. Kooiman, J.: Governing as Governance. Sage Publications, London (2003)

17. Krook, M.L., True, J.: Rethinking the Life Cycles of International Norms: The United Nations and the global promotion of gender equality. European Journal of International Relations $\mathrm{XX}(\mathrm{X}), 1-25(2010)$

18. Monge, P.R., Contractor, N.S.: Theories of Communication Networks. Oxford University Press, Oxford (2003)

19. Onuf, N.: World of Our Making: Rules and Rule in Social Theory and International Relations. University of South Carolina Press, Columbia (1989)

20. Padovani, C., Pavan, E.: Actors and Interactions in Global Communication Governance: The Heuristic Potential of a Network Approach. In: Mansell, R., Raboy, M. (eds.) The Handbook of Global Media and Communication Policy, pp. 543-563. Blackwell, Oxford (2011)

21. Pavan, E.: Frames and Connections in the Governance of Global Communications. A Network Study of the Internet Governance Forum. Lexington Books, Laham (2012)

22. Raboy, M., Padovani, C.: Mapping Global Media Policy: Concepts, Frameworks, Methods. Communication, Culture and Critique 3(2), 150-169 (2010)

23. Sending, O.J.: Norms, Knowledge and Constitutive Relations. Paper presented at the ISA Annual Convention, San Diego, March 22-25 (2006)

24. Vespignani, A.: Predicting the Behavior of Techno-Social Systems. Science 325, 425-430 (2009) 